\title{
tic\&société
}

Vol. 12, $\mathrm{N}^{\circ} 1$ | 1er semestre 2018

Communs numériques et communs de la connaissance

\section{Une introduction aux communs de la connaissance}

\section{Hervé LE CROSNIER}

\section{(2) OpenEdition}

Journals

Édition électronique

URL : http://journals.openedition.org/ticetsociete/2481

DOI : 10.4000/ticetsociete.2481

\section{Éditeur}

Association ARTIC

Édition imprimée

Pagination : 13-41

\section{Référence électronique}

Hervé LE CROSNIER, "Une introduction aux communs de la connaissance », tic\&société [En ligne], Vol. $12, N^{\circ} 1$ | 1er semestre 2018, mis en ligne le 31 mai 2018, consulté le 09 octobre 2020. URL : http:// journals.openedition.org/ticetsociete/2481 ; DOI : https://doi.org/10.4000/ticetsociete.2481 
tic\&socété - 12(1), 2018

\section{Une introduction aux communs de la connaissance}

\section{Hervé LE CROSNIER}

Hervé Le Crosnier est éditeur multimédia chez C\&F éditions. II était auparavant conservateur de bibliothèque et fondateur de la liste de diffusion Biblio-fr, puis enseignant chercheur sur les technologies du web et la culture numérique à l'Université de Caen-Normandie. herve.lecrosnier@zerlo.fr 


\section{Une introduction aux communs de la connaissance}

Résumé : Nous proposons une introduction aux communs de la connaissance qui s'appuie sur une tentative de définir les communs eux-mêmes, pour évaluer ensuite ce qui s'y rapporte dans les domaines intangibles du savoir et du numérique. II apparaît difficile d'avoir une définition canonique des communs. Malgré un corpus scientifique en augmentation rapide, il subsiste des approches différentes selon les pays, les régions, les cultures... Mais cela est certainement un bienfait: les communs sont avant tout le résultat d'une expérience vécue. L'article présente différentes approches des communs, à la fois dans les débats théoriques et dans les pratiques des mouvements associés au partage de ressources. II documente le passage d'une théorie appliquée à des ressources localisées vers des pratiques coopératives élargies grâce au numérique.

Mots-clés: communs, biens communs, enclosures, publications scientifiques, accès libre, Creative Commons, logiciels libres, Elinor Ostrom.

Abstract: We propose an introduction to the knowledge commons based on an attempt to define the commons themselves, and then evaluate how they are related to the intangible fields of knowledge and the digital. It seems difficult to settle on a canonical definition of the commons. Despite a rapidly growing scientific corpus, there are still different approaches according to countries, regions, cultures... But this is certainly a benefit: the commons are above all the result of lived experience. The article presents different approaches to the commons, both in theoretical debates and in the practices of movements associated with resource sharing. It documents the shift from theory mainly concerned with localized resources to cooperative practices expanded through digital technology.

Keywords: commons, common goods, enclosures, scientific publications, open access, Creative Commons, free software, Elinor Ostrom. 
Resumen: Se propone una introducción a los bienes comunes del conocimiento, basada en un intento de definir los bienes comunes, y en la evaluación de la relación de estos con los campos intangibles del conocimiento y lo digital. No resulta fácil establecer una definición canónica de los bienes comunes. A pesar del rápido crecimiento del corpus científico, todavía existen diferentes enfoques, según los países, las regiones, y las diferentes culturas.... Esta diversidad resulta beneficiosa ya que los bienes comunes ante todo son el resultado de una experiencia vivida. El texto presenta diferentes enfoques de los bienes comunes, tanto en los debates teóricos como en las prácticas de los movimientos asociados con el intercambio de recursos. A la vez, analiza el paso de una teoría aplicada a los recursos localizados hacia determinadas prácticas cooperativas, posibilitadas por la tecnología digital. Finalmente, se propone una bibliografía que permite profundizar en esta introducción a los bienes comunes.

Palabras claves: bienes comunes, vallados, publicaciones científicas, acceso abierto, Creative Commons, software libre, Elinor Ostrom 
Alors que les références aux communs ou au bien commun se multiplient, souvent dans un usage racoleur et prêtant à confusion que l'on nomme le commons washing, l'approche scientifique de ce que recouvrent ces deux termes, et d'autres construits autour des mêmes racines, reste complexe et débattue. Dans l'approche traditionnelle, les communs sont des structures sociales localisées, appuyées sur des ressources naturelles: pêcheries, forêts, réseaux d'irrigation, marais entretenus collectivement, etc. Les "communs urbains " forment une première approche qui se détache de ce corps de doctrine, puisque la propriété publique est concernée en même temps que l'activité autonome des habitants. Mais étendre la notion à des biens de très grande taille, d'une part, et à la connaissance et aux autres formes immatérielles, d'autre part, relève de débats importants. Pour notre part, nous pensons que les communs sont, d'un côté, ce que nous avons ensemble, ce dont nous héritons et qu'il nous faut maintenir et préserver pour les générations futures et, d'un autre côté, ce que nous construisons ensemble dans l'esprit de partage. Ces deux approches sont certes différentes, mais les ressorts à l'œuvre dans l'activité sociale de construction et de défense de ces communs restent semblables, car parler des communs, bien au-delà de la ressource considérée, c'est avant tout parler d'une forme d'organisation sociale, d'arrangements institutionnels, de construction collective, d'autogestion et, bien évidemment, de partage, et donc d'une nouvelle relation à l'autre.

\section{Du foncier au savoir : une extension appuyée sur la théorie}

L'extension de la notion de communs du local au global et à l'intangible suit le parcours d'Elinor Ostrom, certainement la chercheuse la plus connue dans le champ de recherche consacré aux communs. Elinor Ostrom a obtenu en 2009 le prix de la Banque de Suède en sciences économiques (communément appelé le "Nobel d'économie ") pour ses travaux sur les communs (Le Crosnier, 2011b). Elle a commencé dans les années 1960 en étudiant les réseaux 
Une introduction aux communs de la connaissance

d'irrigation de la Californie du Sud, pour y repérer les arrangements institutionnels que mettaient en place les appropriateurs (soit, dans le langage scientifique associé aux communs, les personnes concernées par l'usage du commun) pour assurer la maintenance de ces outils collectifs indispensables à l'activité privée de chaque agriculteur. Quand en décembre 1968 Garrett Hardin publie son célèbre article "La tragédie des communs » (Hardin, 1968), croyant enterrer ainsi la notion de communs, il va au contraire provoquer un rebond de la part des chercheurs engagés dans l'étude réelle des communs agricoles et fonciers, au premier chef Vincent et Elinor Ostrom, et engendrer ce que l'on nommera l'École de Bloomington.

La fable d'Hardin consiste à dire que, dans un champ donné et ouvert à tous, l'intérêt personnel des éleveurs est de placer le plus grand nombre de bêtes... ce qui va conduire à un surpâturage et finalement " à la ruine de tous ». Or jamais dans l'étude des situations réelles les chercheurs de Bloomington n'ont observé de telles situations. Au contraire, les usagers discutent des règles à appliquer pour protéger la ressource qu'ils partagent (common pool ressource). D'ailleurs, comme nous le rappelle Alain Rey (2011), "communs » et " communication" ont la même racine latine. Les travaux d'Ostrom donneront lieu à la création de l'IASC (International association for the Study of Commons) et à la publication du livre majeur Governing the commons ${ }^{1}$ en 1990 (Ostrom, 1990). Ce n'est qu'avec le développement de l'Internet que la notion de communs appliquée au numérique va connaître un second souffle, qui conduira Elinor Ostrom à coordonner, en 2007, l'ouvrage Understanding knowledge as a commons, qui synthétisera les idées portées par les tenants du numérique dans le cadre scientifique des communs (logiciels libres, accès libre à la science, rôle des brevets...) (Hess et Ostrom, 2007). Enfin, peu avant son décès survenu en 2012, au moment même de $\mathrm{RIO}+20$, la rencontre multilatérale sur la protection de la biodiversité tenue en 2012, vingt ans après la première conférence de Rio sur ce thème, un des derniers textes d'Elinor Ostrom (2009) sera consacré à l'approche du climat sous

1 Traduit en français en 2010: Ostrom, E. (2010). La gouvernance des biens communs: pour une nouvelle approche des ressources naturelles. Bruxelles, Belgique : De Boeck. 
l'angle des communs, mettant notamment en avant la notion de gouvernance polycentrique: il ne faut pas attendre la décision prise à une échelle mondiale pour commencer à traiter d'un problème global ; il y a des responsabilités emboîtées et des actions de défense des communs qui se mobilisent à chaque niveau, de l'individu engagé dans une démarche de buen vivir ("vivre mieux avec moins", suivant le message des communautés traditionnelles andines), aux villes en transition et aux villes résilientes, en passant par les États qui définissent des objectifs de réduction carbone, jusqu'au niveau multilatéral (comme ce qui deviendra l'Accord de Paris).

Malgré les réticences de certains chercheurs (Greer, 2015²) qui préfèrent voir l'étude des communs centrée sur des " communaux », des systèmes locaux d'entraide et de gestion collective autour de ressources physiques partagées, la notion mérite d'être effectivement étendue plus largement. Dans Governing the commons, Elinor Ostrom relève, à partir de ses enquêtes de terrain, huit principes associés à des communs ayant réussi et qui portent sur le caractère local de la ressource et des communautés concernées. Des critères de succès qui n'ont pas empêché son auteure elle-même d'en élargir la portée. Car le climat comme le numérique, dans leurs usages et leurs conséquences, sont en fait partout locaux et en même temps gérés et organisés de façon globale, d'où l'importance des responsabilités emboîtées telle qu'elles figurent dans ces huit principes :

- des groupes aux frontières définies ;

- des règles régissant l'usage des biens collectifs qui répondent aux besoins et aux spécificités locales ;

- la capacité des individus concernés à modifier ces règles ;

- le respect de ces règles par les autorités extérieures ;

- le contrôle du respect des règles par la communauté ;

- un système de sanctions graduées ;

\footnotetext{
${ }^{2}$ Voir également la réponse apportée par Lionel Maurel dans la même revue : Maurel, L. (2015, 29 septembre). "Communs de la connaissance et enclosures». La vie des idées. Repéré à http://www.laviedesidees.fr/Communs-de-laconnaissance-et-enclosures.html
} 
Une introduction aux communs de la connaissance

- l'accès à des mécanismes de résolution des conflits peu coûteux ;

- la résolution des conflits et les activités de gouvernance organisées en strates différentes et imbriquées.

On voit que ces principes forment une analyse « institutionnelle »: comment les actrices et les acteurs des communs vont-ils trouver les moyens de faire perdurer leur association autour d'une ressource? Comment garantir que les décisions, en général prises par consensus, vont néanmoins être appliquées ? On voit ici que les communs ne constituent pas une utopie abstraite, mais prennent bien en compte les difficultés des arrangements entre individus, surtout dans les sociétés contemporaines, où le marché des biens d'une part et la propriété publique de l'autre sont les formes dominantes et influencent donc l'ensemble des réflexions.

Les applications sont parfois difficiles, comme le montre Nolwenn Weiler (2016) à propos des marais de Guérande. Dans cet exemple, le commun se situe dans l'organisation du marais (réfection des buttes d'argiles, circuit de l'eau de mer) et de l'entraide, alors que les parcelles, la récolte de sel et la commercialisation sont le fait de paludiers privés, montrant que les communs peuvent aussi s'accorder avec diverses formes de propriété et de marché, car ils traitent principalement de l'usage des biens. Cependant, comme dans toute construction sociale, les frictions peuvent toujours intervenir, comme lorsqu'il a fallu décider d'empêcher l'eau de mer d'entrer dans le marais suite au naufrage du pétrolier Amoco-Cadiz. Une année blanche est difficile pour un paludier isolé ; maintenir la qualité du marais est important pour la collectivité des paludiers. Cette contradiction montre l'importance des règles institutionnelles mises en lumière par Elinor Ostrom. II a fallu que des rondes s'organisent (faire respecter les décisions) pour garantir qu'une minorité ne pourrait pas ouvrir les vannes, et il a fallu offrir des aides pour que les plus touchés puissent attendre une année.

Cependant, une lecture élargie de ces critères et de ces principes peut rendre compte de types de biens différents, soit immatériels soit globaux. Avec Mélanie Dulong de Rosnay (Dulong de Rosnay, Le Crosnier, 2012), nous avons montré comment ces huit principes se retrouvaient dès lors que l'on considérait l'Internet comme un commun. 


\section{Approches différenciées des communs dans le monde}

À côté des débats théoriques portant sur l'extension de la notion de communs au-delà des initiatives localisées, nous avons assisté, depuis le milieu des années 2000, à la naissance et au développement d'un «mouvement des communs"; non pas une structure formalisée, mais des dynamiques, des actions, des pratiques qui se réfèrent à la notion de commun. Bien évidemment, ces activités collectives existent depuis longtemps, et ce, indépendamment de la théorie, en témoignent deux films documentaires rapportant des expériences appartenant à la sphère d'activité des communs, même si le terme lui-même n'est jamais prononcé : Demain (2015), de Cyril Dion et Mélanie Laurent, et Nul homme n'est une île (2018), de Dominique Marchais. D'un certain point de vue, comme nous organisons une large part de notre vie collectivement, nous sommes toutes et tous des pratiquants ordinaires des communs. Or, souvent, nous n'assimilons pas nos pratiques, ou celles que nous observons, à des communs. II faut un long jeu de va-et-vient entre la théorie, les publications et la capacité des acteurs à se reconnaître comme éléments d'une dynamique collective qui dépasse chaque activité propre.

Le fait de réunir diverses pratiques collectives sous l'ombrelle des communs est un double processus : d'une part, la théorie accueillante des communs, comme nous l'avons montré ci-dessus, se développe ; d'autre part, les mouvements ont besoin de termes dans lesquels ils peuvent se reconnaître, construire une parenté, afin de pousser les idées qu'ils portent plus globalement et agir sur les normes culturelles d'une époque. II est intéressant de voir comment la notion de communs a été introduite dans le cadre du Forum social mondial (FSM) de 2009 à Belém au Brésil (Ambrosi, 2010). C'est par l'intermédiaire du Forum mondial «sciences et démocratie " que des chercheurs et des associations engagées sur le terrain scientifique ont présenté ce concept, l'ont lié à l'activité traditionnelle de la recherche et ont plaidé pour les modèles d'ouverture, comme le logiciel libre ou l'accès libre aux publications. Intéressés, les animateurs du FSM ont quelques jours après proposé un manifeste rédigé par Frédéric Sultan et José Correa "pour la récupération des biens communs" (http://bienscommuns.org/), alors même que le terme n'avait 
jamais été utilisé auparavant par ces mouvements sociaux altermondialistes. Trois ans plus tard, le Sommet des peuples, qui se tenait en parallèle de la rencontre multilatérale $\mathrm{RIO}+20$, avait comme intitulé «Pour la justice sociale et environnementale en défense des biens communs "(Chartier et Le Crosnier, 2015). Cette montée du thème des communs se retrouve en France, notamment lors de deux semaines coordonnées de défense et d'illustration des communs (Villes en biens communs en 2013 et Le temps des communs en 2015). Ces initiatives ont réussi à se faire connaître et à faire reconnaître des activités éparses comme parties prenantes d'un mouvement plus global, partageant des conceptions et des méthodes communes. En 2018 s'est ouvert à Paris une "Maison du libre et des communs", alors que des "Assemblées des communs » ont eu lieu dans plusieurs villes françaises (Brest, Lille, Grenoble) et se sont fédérées entre elles. On le voit à travers ces quelques exemples, les pas sont rapides.

Toutefois, la notion même de commun, qui est une notion théorique, mais également une notion utilisée spontanément, s'incrustant rapidement dans le langage de tous les jours, n'a pas la même signification, ni pour tous les auteurs ni pour les mouvements émergeant dans divers pays.

Ainsi, en Amérique latine, les communs renvoient à la nature globale et à la biodiversité. II s'agit d'une approche qui prend ses racines dans la double dimension d'une résistance à l'extractivisme et d'une montée des revendications des peuples autochtones. C'est ainsi que les communs y rencontrent les notions de Pachamama (Terre-Mère, ou Terre nourricière) et de buen vivir. Pablo Solon, alors qu'il était ambassadeur de la Bolivie auprès de l'ONU, a œuvré pour laïciser la notion de Terre-Mère et obtenir qu'il existe un " Jour de la Terre-Mère » (International Mother Earth Day). Depuis 2009, le 22 avril de chaque année permet de rappeler que la Terre-Mère pourrait devenir actrice juridique et porter devant les tribunaux les atteintes à ses droits fondamentaux. La notion de communs est également fortement associée à celle de coopératives de services collectifs, comme le montre l'exemple de Cecosesola (2015), qui réunit plusieurs dizaines de milliers de personnes au Venezuela. 
Le travail juridique consistant à donner des droits à des entités non humaines est novateur et rejoint la nécessité de lutter contre l'« écocide " que nous vivons. En 2017, le parlement néo-zélandais a ainsi doté de la «personnalité juridique » le fleuve Whanganui et le Mont Taranaki, mis sous la protection juridique de plusieurs communautés maories.

En Afrique, la notion de communs est largement utilisée pour représenter les modes d'usage de la terre par les villageois, en dehors de la notion de propriété, et cela au moment même ou l'accaparement des terres par des États (notamment asiatiques) ou des entreprises multinationales vient profondément modifier les modes traditionnels de partage de la terre entre paysans, mais aussi entre paysans et éleveurs, entre sédentaires et nomades (Le Roy, 2015).

Aux États-Unis, la notion de communs est souvent très proche de celle de domaine public, que ce soit pour la domanialité publique (parcs nationaux, plages, espace urbain...) ou intellectuelle. Elle s'intègre dans la pensée de ce pays qui cherche souvent à calculer l'optimum économique dans l'usage et la propriété d'une ressource. Yochai Benkler (2006) développe ainsi la notion de common-based peer production pour souligner que la dynamique des réseaux et du numérique permet d'organiser la collaboration sur une échelle étendue et d'ainsi produire à moindre coût des services impensables dans un régime commercial, comme l'encyclopédie Wikipédia, ou le site d'échange d'information pour les programmeurs Slashdot. Quand il écrit le premier ouvrage concernant les communs de la connaissance, le juriste James Boyle (2008) l'intitule The Public Domain.

En élargissant le concept de production de pair à pair, Michel Bauwens, en travaillant avec plusieurs pays et villes (Équateur, Ville de Gand en Belgique...) montre que les pratiques collaboratives existent et peuvent former un levier pour changer radicalement la conception de la production et de l'échange. II propose, à partir de ces études de cas, des perspectives s'appuyant sur les communs pour sortir du cycle actuel de conjonction des crises économiques et de sociabilité dans une logique postcapitaliste (Bauwens et Kostakis, 2017).

Au Québec, le "printemps érable » a mobilisé les étudiants autour de l'éducation comme bien commun, une manière de 
dire que l'intérêt des populations est de conserver un service public de l'éducation, au prix abordable et ouvert sur les usagers, les enseignants et les étudiants.

En Espagne, alors que plusieurs municipalités se réclament des communs (Barcelone, Madrid...), c'est principalement la question urbaine qui est centrale: logement, occupation de l'espace public (qui est interdit en Espagne par une loi récente suite aux mouvements de mai 2011). Les communs sont ainsi souvent associés au nouveau municipalisme (Cillero, 2016). La Catalogne est également l'endroit où se construit la $\mathrm{CIC}$, Coopérative intégrale de Catalogne, qui tente de reconstruire un monde " en communs » à l'intérieur du système tel qu'il est, regroupant des services, des artisans, des individus, et développant des moyens d'échanges (monnaies affectées) et des modes de vie collective (Dafermos, 2017).

Cela dit, c'est certainement en Italie que la question des communs a été le plus développée, en pratique comme en théorie, et s'est inscrite dans de nombreux textes des municipalités (Lucarelli, 2018). En 2007, le juriste Stefano Rodotà crée une commission pour rédiger un addendum à la Constitution italienne afin de faire prendre en charge des " biens communs" (beni comuni) en les considérant comme des «biens qui expriment une utilité fonctionnelle pour l'exercice des droits fondamentaux et le libre développement de la personne ॥ (Festa, 2016). Si le Sénat n'a pas validé officiellement le travail de la commission, l'idée a fait son chemin dans les populations. Le référendum contre la privatisation de l'eau de 2011 et, la même année, l'occupation de théâtres pour tenir des lieux autonomes de débat et d'activité culturelle ont montré l'importance de cette notion. Des villes comme Naples ou Bologne ont établi des chartes pour laisser une partie de l'activité directement aux mains des habitants, organisant des partenariats public-communs « sur la collaboration entre les citoyens et l'administration pour l'entretien et la régénération des biens communs urbains ${ }^{3}$ » (Communa di Bologne, 2013, p. 1).

\footnotetext{
${ }^{3}$ Traduction libre de : « sulla collaborazione tra cittadini e amministrazione per la cura e la rigenerazione dei beni comuni urbani ».
} 


\section{Une définition complexe}

On voit ainsi que la notion de communs reste difficile à définir, qu'elle est abordée différemment selon les histoires théoriques, mais aussi selon les traditions culturelles, mais on sent bien qu'il y a une homogénéité dans le projet: rendre la main aux acteurs eux-mêmes et agir par le droit pour stabiliser et renforcer les pratiques émanant des populations. Ce rôle central du droit est important, car, dans la logique des communs, il est élaboré "par en bas ». David Bollier, Michel Bauwens et Stacco Troncoso (2015) ont ainsi repéré des formulations juridiques venant des acteurs des communs et les ont regroupées dans un site "Law for the Commons". De ce point de vue, les deux formes juridiques de référence sont constituées autour de la connaissance : la GPL (General public licence), pour le logiciel libre, et les licences Creative Commons, pour les œuvres culturelles.

L'école française autour des communs, particulièrement représentée par le Dictionnaire des biens communs paru en 2017 (Cornu, Orsi et Rochfeld, 2017), définit les communs autour de trois axes :

- Le type de ressource (ou de service) mis en partage. On doit évidemment distinguer les biens épuisables et rivaux, pour lesquels l'usage de l'un va limiter celui de l'autre, et les biens immatériels, qui, au contraire, prennent de la valeur quand un grand nombre de personnes peuvent s'en emparer.

- La communauté qui va prendre en charge cette ressource et maintenir le partage ouvert. Une telle communauté peut être restreinte (les parents d'une crèche parentale) ou plus large (les peuples autochtones que l'on charge généralement de la maintenance de la biodiversité). Cependant, à la différence des communautés de destin, régies par le lieu de naissance ou la religion, il s'agit ici en général de communautés de choix, construites autour de l'activité volontaire. Une même personne peut ainsi appartenir à plusieurs communautés de communs.

- Les règles de droit qui vont s'appliquer, en interne parmi les acteurs du commun considéré et vers l'extérieur pour les usages ponctuels. Dans ce cadre, la notion de 
Une introduction aux communs de la connaissance

faisceau de droit (Orsi, 2013), qui distingue les diverses parties habituellement liées à la propriété, représente une avancée juridique, par exemple exploitée par les community land trust (habitat collectif) ou par les licences Creative Commons (plusieurs droits ouverts en fonction de la volonté de l'auteur).

On doit ajouter à ce triptyque la question des enclosures : qu'est-ce qui met en danger l'accès aux communs et leur usage ? Cela d'autant plus que nous savons que les gens ne mesurent pas toujours ce dont ils disposent et qui est par ailleurs gratuit, ou invendable, et se rendent souvent compte de son importance quand on essaie de leur enlever. Les formes d'enclosure sont multiples et très diversifiées (Le Crosnier, 2011a), allant de la privatisation pure et simple à des règles juridiques limitant le partage (les multiples extensions de la propriété intellectuelle depuis une trentaine d'années) ou à des moyens techniques interdisant la circulation (DRM [Digital Rights Management systems] pour les fichiers numériques ou GURT [Genetic Use Restriction Technologies] pour les plantes).

Dans cette approche, le « bien », au sens économique, n'est que le prétexte à une construction sociale de communauté, avec toutes les difficultés et les engagements que cela nécessite. En ce sens, les communs sont également une école de démocratie active, notamment parce que règne la " doocracy ", c'est-à-dire le pouvoir des agissants : ceux qui vont faire les choses et mettre en place le commun vont avoir plus d'importance dans la gouvernance communautaire. C'est ce que l'Internet résume dans la devise « rough consensus and running code ».

\section{Questions de vocabulaire}

On peut, à la lumière de cette approche, mesurer les différences, mais aussi les convergences entre les divers termes utilisés pour parler des communs.

Dire les «biens communs " renvoie principalement à l'approche économique, centrée sur la ressource et sa propriété. Quand on veut parler de l'organisation sociale, d'une approche centrée sur la communauté et sa gouvernance, on 
utilise en général le terme de "communs " au pluriel, même si cela n'est pas toujours facile d'un point de vue grammatical. Si on veut élargir encore et invoquer le "commun » au singulier, il s'agit alors de définir un principe démocratique, une forme instituante qui va interroger l'organisation sociale en général (Dardot et Laval, 2014).

D'un point de vue plus théologique, on pourrait même dire téléologique (guidé par les finalités), "le bien commun» apparaît comme une destination à atteindre, mais qui reste suffisamment vague, non rattachée à telle ou telle ressource ou tel ou tel service concret. L'expression est de ce fait malheureusement utilisée comme une lointaine récompense, mobilisée pour inciter les populations à assumer les difficultés actuelles. En ce sens, le bien commun, auquel nul ne peut s'opposer en raison même de son caractère intemporel, se distingue de l'intérêt général, qui est le sel de la politique et se décide par un débat ouvert devant le « tribunal de la raison » et parfois plus prosaïquement devant des tribunaux civils ou ad hoc, comme le Tribunal Russel, le Tribunal sur Monsanto, et d'autres activités qui mettent en scène un procès des enclosures au nom de la défense d'un intérêt général applicable dès maintenant. Du point de vue des communs, il s'agit alors de définir les porteurs des responsabilités associées et ayant la capacité à ester en justice au nom de cet intérêt général, une question que l'on retrouve pour déterminer quels pourraient être les représentants légaux des "générations futures », ou du « patrimoine mondial de l'humanité ».

On trouve également le terme de bien public, qui correspond à une approche économique renvoyant aux travaux de Paul A. Samuelson (Samuelson, 1954 ${ }^{4}$ ) distinguant des biens non rivaux (l'usage de l'un n'obère pas celui par l'autre) et non excluables (on ne peut en empêcher l'usage). On a souvent utilisé cette formule dans les années 1990 pour désigner les fichiers numériques avant de comprendre qu'au contraire, ceuxci pouvaient également être l'enjeu d'enclosures et que les verrous internes (DRM), la perte de références et de sauvegardes ou les règles juridiques pouvaient limiter la

\footnotetext{
${ }^{4}$ Une traduction en français de son article est proposée dans l'ouvrage de Jacques Généreux: J. Généreux (2006). L'économie politique. Analyse économique des choix publics et de la vie politique. Paris, Franc : Larousse.
} 
capacité proprement « matérielle » du numérique à dupliquer et à faire circuler les documents (Hess, 2011).

\section{Domaine public}

Enfin, on ne saurait parler des termes désignant tel ou tel aspect des communs sans évoquer la notion de domaine public, qu'il soit foncier, urbain ou intellectuel. C'est souvent sur le domaine public que se construisent des communautés de partage. Pour autant, deux siècles d'étatisation ont construit une superstructure administrative qui se sent « propriétaire » du domaine public, alors qu'en droit, ces institutions n'en sont que les gestionnaires. Et c'est souvent pour s'opposer aux volontés de cette administration de transformer des lieux du domaine public en biens privés (en général pour construire des centres commerciaux) que les gens se sont mobilisés, notamment dans ce qu'on a appelé le mouvement des places (Istanbul, parc Gezi ; Occupy à New York, parc Zuccoti...).

En matière de biens culturels, on parle ainsi de " copyfraud » (Mazzone, 2011) pour désigner l'accaparement par une institution d'œuvres appartenant au domaine public, une occasion en général ouverte par la numérisation de cette œuvre dont certains estiment qu'elle apporterait de nouveaux droits de propriété intellectuelle sur la copie numérique. Cette interprétation est remise en cause par les mouvements comme par les personnalités. On a vu, ainsi, la ministre de la Culture française Fleur Pellerin prendre ostensiblement en photo une œuvre du Musée d'Orsay et la publier sur Instagram, ce que le musée interdisait, et ouvrir ainsi un débat sur le droit de chacun de photographier et de diffuser les œuvres du domaine public (Combis-Schlumberger, 2015). En fait, à côté de l'évolution de mentalité des institutions, c'est quand les acteurs concernés se mobilisent pour utiliser et diffuser le domaine public que l'on développe pleinement des communs : l'action Museomix pour la diffusion de reproductions des œuvres des musées (http://museomix.org); l'action des nombreux éditeurs qui s'appuient sur le domaine public pour sa réhabilitation; ou l'action de SavoirCom1, qui réalise chaque année un " calendrier de l'avent du domaine public » pour présenter les auteurs, peintres, musiciens, etc., qui vont entrer dans le domaine public au $1^{\mathrm{er}}$ janvier de chaque année (SavoirCom1, 
$2017^{5}$ ). A contrario, on voit se développer des règles et des pratiques qui vont limiter l'usage du domaine public architectural: il est devenu impossible d'utiliser une photographie de monuments du patrimoine (Loi « création » de 2016 en France, malgré une décision contraire du Conseil Constitutionnel [Calimaq, 2018]) ou d'obtenir un "droit de panorama " pour inscrire dans des documents publics l'environnement matériel dans lequel nous vivons (https://libertedepanorama.fr).

II reste également une question portant sur la définition du domaine public intellectuel. En dehors du domaine public temporel (soixante-dix ans après le décès d'un auteur), l'association Communia propose une définition élargie : "Le domaine public, tel que nous l'entendons, est constitué de toute la richesse des informations qui sont accessibles et utilisables sans les obstacles habituellement associés à la protection par le droit d'auteur et le copyright ${ }^{6} »($ Communia, 2010, s. p.). Cela ouvre la porte à considérer comme appartenant au domaine public les œuvres sous licence libre, ce que le juriste Michel Vivant appelle le « domaine public consenti ».

\section{Diffuser la connaissance}

Qu'entendons-nous par "connaissance » dans ce contexte ? Les premières études portaient sur les communs issus des pratiques liées à la nature (du glanage à l'organisation coopérative du foncier). Les communs historiques sont souvent assimilés aux espaces partagés (les communaux, les alpages, les marais, la zone de pêche littorale, etc.) et à leur mode de gestion et d'entretien collectif. On sait bien que la nature est fortement affectée par les modes d'organisation, les expériences et les travaux humains issus des sphères immatérielles. La distinction porte donc sur les activités spécifiques qui relèvent de la mise en forme des réflexions, sur la production documentaire. La connaissance apparaît comme le terme générique pour désigner ce qui ne ressort justement pas d'une accroche à un espace localisé. Les « connaissances

\footnotetext{
${ }^{5}$ Voir également : Taffin, N. (2014) Pages publiques : à la recherche des trésors du domaine public. Caen, France : C\&F éditions.

${ }^{6}$ Traduction libre de: "The public domain, as we understand it, is the wealth of information that is free from the barriers to access or reuse usually associated with copyright protection ».
} 
traditionnelles ", d'une part, et les modèles de gestion coutumiers, d'autre part, forment l'univers des savoirs associés de longue date à l'usage de la nature. Mais d'autres formes de connaissances sont dorénavant liées aux espaces naturels et au comportement du vivant: depuis la cartographie jusqu'à l'analyse du génome, chaque élément de la nature est porteur de connaissances. La gestion commune de la nature passe par le partage de l'usage de ces savoirs, que ce soit pour l'agriculture, la médecine ou les sciences humaines. Nous vivons dans une "société de la connaissance ", pour l'instant largement dominée par une " économie de la connaissance ", dans laquelle les savoirs deviennent des enjeux de pouvoir et de richesse.

Nous considérons ici la connaissance comme la production de supports de référence pour le savoir et la culture et nous cherchons à étudier à la fois les freins et les incitations à partager ces documents et les connaissances qu'ils enregistrent. C'est une vision plus limitée, moins philosophique que ce que contient en général le terme de connaissance, mais qui répond à la manière dont les communs peuvent participer aux objectifs de la société : la transmission (école, livre, bibliothèque...), la production de nouveaux savoirs (science ouverte et participative...) et l'innovation (outils techniques, logiciels, fab labs...). La question qui se pose est de savoir si la construction de communs de la connaissance va apporter de meilleurs résultats dans ces domaines que l'individualisation du savoir à l'œuvre dans les brevets, le droit d'auteur ou le mode d'évaluation de la recherche par les citations.

Un des problèmes liés à la multiplication de régimes de propriété intellectuelle est ce que Michael Heller (1998) appelle la tragédie des anticommuns. Trop de règles de propriété éclatées entre de nombreux acteurs conduisent à sous-utiliser le savoir, à limiter sa circulation. C'est par exemple ce que, dans le domaine de la propriété industrielle, on appelle le " buisson de brevets » : de nombreux actes sont déposés pour couvrir tout un domaine de façon à ce qu'un nouvel entrant soit obligé d'empiéter sur une des propriétés, ce qui ne peut se résoudre qu'entre acteurs importants, chacun disposant d'un portefeuille étendu de brevets qui constitue autant de menaces pour les concurrents qui doivent donc négocier entre partenaires de taille comparable (Lévêque et Ménière, 2003). 
De même, les restrictions portées sur la réutilisation du domaine public empêchent de nombreux travaux d'être réédités ou portés à la connaissance de nouveaux publics. II en est de même du droit de l'audiovisuel, très complexe, qui distingue un droit de l'enregistrement (qui s'évanouit soixante-dix ans après l'enregistrement) et le droit des auteurs, des compositeurs et des réalisateurs (qui perdure jusqu'à soixante-dix ans après le décès du dernier auteur).

La question des communs de la connaissance est également différente de celle de l'accès aux documents. Pour garder son efficacité, la connaissance doit pouvoir s'étendre en permanence, ce qui va au-delà de l'accès, qui, par exemple, est depuis longtemps rendu possible par les bibliothèques. II s'agit de prévoir, dans les règles de droit comme dans les formes du partage une extension du savoir lui-même, mais également une extension du nombre de personnes susceptibles d'avoir accès à ce savoir (droit de rediffusion et de réédition). La gouvernance des communautés scientifiques (université) ou épistémiques (Wikipédia) porte donc sur la double évaluation : de la crédibilité des propositions et de la capacité à les faire circuler. C'est la question qui est posée aux tenants de l'accès libre aux publications scientifiques, qui, souvent, souhaitent remettre sur le métier le mode d'évaluation scientifique luimême. Parmi les expériences en ce sens, on voit apparaître la notion de preprint publié, soumis à l'intervention collective pour améliorer le document qui sera ensuite inscrit au sommaire d'une revue. Cette expérience a été tentée par la revue Nature et a montré les difficultés de passer d'un modèle de carrière appuyé sur des travaux individuels et secrets jusqu'à leur parution vers un modèle plus collaboratif et ouvert (Nature, 2006).

Le monde scientifique a beaucoup changé depuis la période du milieu du $20^{\mathrm{e}}$ siècle quand le sociologue des sciences Robert K. Merton pouvait repérer des comportements " communalistes " dans le fonctionnement des chercheurs et des laboratoires. Le partage des réflexions, même inabouties, était alors la norme sociale, renforcée notamment par l'effort de guerre et l'interdisciplinarité nécessaire. Or, dans les années 1960, Eugene Garfield invente la scientométrie, qui deviendra rapidement le modèle d'évaluation des chercheurs par les citations. Puis, dans les années 1980, aux États-Unis 
d'abord suite au Bayh-Dole Act, les universitaires vont pouvoir déposer des brevets et jouer un double rôle, à la fois chercheurs publics et chercheurs préparant la mise en place d'entreprises privées commercialisant les recherches. Voilà une situation qui a généré des effets de bords négatifs, particulièrement dans le domaine médical (Leaf, 2005), sans compter la perte de compétences indépendantes dont souffrent aujourd'hui les instances d'évaluation des médicaments et de la santé publique.

II faudra attendre les années 1990 et l'usage répandu de l'Internet dans les universités pour que des chercheurs se référant toujours aux principes collaboratifs de Merton organisent un mouvement pour l'accès libre aux publications. Ce processus a démarré dans la communauté très fermée, mais également très unie, notamment par l'usage des mêmes grands appareils scientifiques, des chercheurs en physique des hautes énergies, avec le service ArXiv créé par Paul Gingsparg en 1992. Ce modèle s'est étendu jusqu'à la publication, en 2002, de I'Initiative de Budapest pour l'accès ouvert (BOAI, 2002), qui va définir l'accès libre et distinguer les deux voies principales de son extension (par le dépôt en archive des prépublications ou par la création de journaux scientifiques diffusés gratuitement). Depuis, ce travail mené par un petit groupe s'est étendu et a rencontré l'intérêt de nombreuses institutions scientifiques. L'impulsion vient d'en bas, de l'action directe de chercheurs, et l'approbation et les moyens complémentaires sont ensuite reconnus et inscrits dans la loi (comme la Loi pour une République numérique en France). II s'agit de ce que l'on peut désigner par le qualificatif de « partenariat public-commun ».

\section{La multiplicité des communs de la connaissance}

Dans le domaine de la création, ce sont les licences Creative Commons (CC) qui marquent une rupture. Un auteur peut, de lui-même, accorder certains droits de réutilisation à ses lecteurs, y compris le droit de modification ou l'abandon du besoin même de citer la provenance avec la licence CC0. Dans l'esprit de ses fondateurs, notamment de Lawrence Lessig (2004), il s'agit de permettre la création d'un vaste répertoire 
d'œuvres culturelles dont la circulation serait assurée par les lecteurs en raison du droit d'usage qui leur est accordé. Les documents numériques ont un coût de reproduction et de diffusion négligeable. Une fois levé le verrou juridique, on pourrait penser que les œuvres sous licence CC vont circuler plus largement, reprises de blogue en blogue. Sauf que depuis, le modèle principal d'utilisation de l'Internet a changé pour passer du blogue ou de la page personnelle à celui des médias sociaux et que la recopie des œuvres pour assurer la rediffusion s'efface devant le lien externe vers la source unique, ce qui limite un peu l'importance des licences CC en les réduisant à un droit d'accès, alors que leur intention était de créer un droit de réédition. Cependant, le nombre d'œuvres étant distribuées sous ce régime montre qu'il existe une réelle attente pour le partage de la culture. Le succès de Wikicommons, qui permet de déposer des photographies ou des vidéos sous une licence Creative Commons, montre bien l'existence d'un désir épistémique parmi la population. L'usage des licences CC, pour les auteurs qui s'y adonnent, va au-delà des règles juridiques. Indiquer volontairement qu'un travail intellectuel est sous licence Creative Commons symbolise une volonté de construire un espace de partage en dehors de la sphère commerciale, en témoigne la révolte des usagers quand le site Flickr a voulu vendre des reproductions imprimées de photographies déposées sous licence CC-by: ils en avaient juridiquement le droit, mais, moralement, ce n'était pas ainsi que les auteurs avaient imaginé le partage (Calimaq, 2014).

On rencontre également profondément ce désir de partager le travail technique dans le domaine du logiciel. La situation est différente: un logiciel n'est jamais fini ; il doit s'adapter aux nouveaux environnements informatiques, les bogues méritent correction. Dès lors, permettre l'accès au code source, au programme tel qu'il est rédigé à l'origine, permet d'améliorer rapidement l'infrastructure technique et à chacun d'ajouter un élément spécifique, ce qui va intéresser les entreprises, qui peuvent disposer d'une base importante, et concentrer leurs efforts sur les spécificités de leur produit ou les demandes de leurs clients. On peut voir, ici, un « partenariat communs-privé » qui permet de rompre la tragédie des anticommuns. Le logiciel lui-même peut circuler et les auteurs (individus ou entreprises), trouver des modèles économiques permettant la poursuite du travail, sauf quand une partie des acteurs ne joue pas le jeu. 
On a pu le voir avec la faille très grave dite " Heartbleed " qui a affecté le logiciel OpenSSL, un élément central de la cryptographie utilisé par la majeure partie des entreprises. La maintenance de ce logiciel, développé par une poignée d'informaticiens qui n'avaient pas assez de moyens (et de temps) pour le maintenir et utilisé par les plus grandes fortunes qui ne songeaient pas à payer pour cela, n'a pas pu s'effectuer dans des conditions correctes, créant ainsi un risque global pour toute l'infrastructure numérique (Kamp, 2014). Cet exemple montre qu'il est dangereux d'assimiler liberté du logiciel et gratuité, celle-ci n'étant qu'un effet secondaire qui ne marche qu'à partir du moment où il circule assez d'argent pour financer les informaticiens qui développent des outils sensibles. C'est aussi ce phénomène qui fait que, de plus en plus, les logiciels libres sont produits par des ingénieurs employés pour cela à plein temps par de grandes entreprises, brisant un mythe du développeur indépendant et montrant à nouveau l'importance de réfléchir à des partenariats (Eghbal, 2017).

La logique des communs de la connaissance s'étend aussi aux données scientifiques et cartographiques. Des licences spécifiques comme l'Open Database Licence permettent de garantir l'ouverture et l'usage d'extraits de bases de données. Elle est notamment utilisée pour la cartographie collaborative, dont le plus important représentant est OpenStreetMap, qui coordonne le travail de milliers de personnes participant à des " cartoparties " pour décrire les points d'intérêt autour de chez eux. Les bases de données des objets célestes, de cartographie du génome, existent et sont largement partagées dans les communautés scientifiques concernées. Cette confiance dans l'ouverture ultérieure des résultats de la recherche incite au développement des sciences participatives, dans lesquelles le public vient fournir des données et participer à l'élaboration scientifique (Le Crosnier, Neubauer et Storup, 2013), une activité que l'on retrouve notamment en agronomie et dans les sciences de l'observation de la nature.

Pour ce qui est de l'éducation et de la transmission, autre versant essentiel des communs de la connaissance, on voit se développer les «Ressources éducatives libres»: les enseignants mettent à disposition des autres enseignants leurs cours et leurs exercices en espérant pouvoir pour d'autres cours profiter des éléments déposés par les autres spécialistes 
de leur discipline. Aussi étonnant que cela puisse paraître, l'éducation n'est pas le secteur des communs de la connaissance qui connaît le développement le plus spectaculaire. Nous en sommes encore à la période où les participants à la production et à l'échange de ressources éducatives libres sont marginaux. L'importance de l'éducation, l'engagement de grandes universités comme le MIT ou d'institutions comme l'Agence universitaire de la Francophonie montre cependant que la reconnaissance ne saurait tarder, audelà de l'UNESCO qui a, dès le début des années 2000, soutenu les initiatives allant dans ce sens. Heureusement, sans attendre les décisions politiques publiques, certains secteurs ayant depuis longtemps une culture du partage pédagogique produisent des matériaux libres, à l'image des mathématiques avec la série de manuels collaboratifs ouverts Sesamath.

D'autres pratiques relevant des communs de la connaissance pourraient être décrites ici, depuis les fab labs, les hackerspaces, l'échange de semences, jusqu'à la création d'œuvres dérivatives réutilisant des extraits d'autres documents (collage en peinture, remix musical, mashup vidéo...).

Dans tous les cas, il s'agit de souligner l'importance de l'Internet et du numérique, vecteur principal de l'extension de ce désir de partage, et de faire vivre les communs de la connaissance. Outil de coordination souple et outil de transaction à faible coût, l'Internet lui-même doit être considéré comme un commun de la connaissance et l'infrastructure permettant tous les autres. Les premiers ingénieurs de l'Internet ont décidé de créer un réseau dont l'évolution, les règles et les normes techniques seraient décidées de manière pleinement transparente et ouverte par les acteurs eux-mêmes. La communauté des ingénieurs, regroupée dans I'IETF (Internet Engineering Task Force) et le W3C (World Wide Web Consortium), définit les protocoles, les usages et assure la fluidité globale de l'Internet, un outil qui est pourtant perclus de propriétés privées, depuis le terminal d'accès qui appartient à l'usager, le serveur appartenant aux services consultés, en passant par le réseau de télécommunication. Ce qui en fait un commun est à chercher dans la définition et l'implémentation ouverte des protocoles qui font fonctionner ensemble tous ces éléments privés, indépendamment des choix de chaque constructeur. Or, comme tout commun, l'Internet est aujourd'hui 
menacé d'enclosures, et cela sur de nombreux fronts, à commencer par la fin programmée de la «neutralité de l'Internet » qui garantit un traitement égal pour chacun, ou par la monopolisation des accès par le biais de machineries publicitaires (moteurs de recherche, médias sociaux, etc.). Une nouvelle défense de l'Internet comme bien commun pourrait s'avérer nécessaire dans les années qui viennent.

\section{Conclusion}

Nous avons essayé, dans cet article d'introduction aux communs de la connaissance, de partir d'une tentative de définir les communs pour évaluer ensuite ce qui s'y rapporte dans les domaines intangibles du savoir et du numérique. II apparaît difficile d'avoir une définition canonique des communs. Malgré un corpus scientifique en augmentation rapide, il subsiste des approches différentes selon les pays, les régions, les cultures... Mais cela est certainement un bienfait: les communs sont avant tout une expérience vécue. Le partage, l'échange non marchand, le don constituent depuis toujours un des socles des sociétés, qui restent pourtant moins reconnu que le marché ou la puissance publique. II existe trois sphères : le marché, la puissance publique et les communs qui, loin d'être séparés et étanches, ont des zones de recouvrement. Malheureusement, les communs restent trop souvent dans l'ombre, la lumière médiatique, financière, politique..., se portant principalement sur les deux autres sphères. On n'a jamais autant parlé de l'Internet que depuis qu'il s'agit de voir la mainmise de quelques groupes marchands utilisant l'économie de l'attention et les données de leurs usagers pour amasser des fortunes insolentes. L'infrastructure ouverte portée par les ingénieurs et les techniciens de l'Internet, reprise par les milliers de développeurs de sites web et les millions d'inconnus qui déposent leur savoir pour le partager, ne fait pas autant recette au billboard du succès médiatique. Le logiciel libre, pourtant indispensable au fonctionnement global du numérique, est toujours réduit à la gratuité, sans qu'on s'attarde aux risques liés à une sous-maintenance provoquée par l'absence de financement par les acteurs privés ou publics... qui pourtant les utilisent. 
Les communs de la connaissance viennent de l'activité autonome de petits groupes qui sont poussés par un désir de partage du savoir et de la culture, mais ils grandissent rapidement. Wikipédia est un des sites les plus visités de la planète. Les États commencent à reconnaître le rôle de l'échange ouvert et à le défendre dans des lois certes timides et parfois concurrencées par d'autres qui accentuent l'enclosure du commun, mais néanmoins significatives d'une nouvelle prise en compte de l'activité coopérative (Peugeot, 2016). Dans le domaine numérique, des alliances inédites entre acteurs privés et du commun montrent qu'il faut avant tout considérer ces derniers au prisme de la formule de Valérie Peugeot: "Les communs sont une utopie pragmatique » (VECAM, 2011).

Les communs sont aujourd'hui le terme qui recouvre des activités très différentes dans leurs objectifs, mais très semblables dans leurs méthodes. II s'agit d'un construit social dont l'enjeu se mesure en capacitation des acteurs, de promesse d'un monde plus solidaire et dans les tentatives de faire exister dès aujourd'hui cet autre monde, sans attendre des lendemains qui chanteraient autour de la prise d'un pouvoir par en haut. II s'agit d'agir et d'échanger, de partager et de bien vivre. II s'agit d'une révolution douce qui grandit au cœur même des éléments les plus avancés du capitalisme cognitif pour le subvertir et faire place à la volonté coopérative et aux espoirs de liberté, d'égalité et de fraternité. II s'agit de nouvelles lunettes permettant de regarder l'avenir autrement.

\section{Références}

Ambrosi, A. (2010). Sciences et démocratie. Caen, France : C\&F éditions.

Bauwens, M. et Kostakis, V. (2017). Manifeste pour une véritable économie collaborative: vers une société des communs. Paris, France: ECLM, 2017. Repéré à http://www.eclm.fr/ouvrage-386.html

Benkler, Y. (2006). The Wealth of Networks: How Social Production Transforms Markets And Freedom. New Haven, CT : Yale UP. Repéré à http://www.benkler.org/Benkler_Wealth_Of_Networks.pdf 
Une introduction aux communs de la connaissance

BOAl. (2002). Budapest Open Archive Initiative. Repéré à http://www.budapestopenaccessinitiative.org/read

Bollier, D., Bauwens, M. et Troncoso, S. (dir.) (2015). Law for the Commons Wiki. Repéré à

http://wiki.commonstransition.org/wiki/Law_for_the_Commons

Boyle, J. (2008). The Public Domain: Enclosing the Commons of the Mind. New Haven, CT : Yale University Press. Repéré à http://thepublicdomain.org/thepublicdomain1.pdf

Calimaq (2014, 29 décembre). Flickr et le chaînon manquant des licences. S.I.Lex. Repéré à

https://scinfolex.com/2014/12/29/flickr-et-le-chainon-manquantdes-licences/

Calimaq (2018, 16 février). Après la décision Chambord, comment sortir d'un domaine public « résiduel »?. S.I.Lex. Repéré à https://scinfolex.com/2018/02/16/apres-la-decisionchambord-comment-sortir-dun-domaine-public-residuel/

Cecosesola (2015). "We Are One Big Conversation": Commoning in Venezuela. An Interview with Members of Cecosesola. Dans D. Bollier et S. Helfrich (dir.), Patterns of commoning. Amherst, MA: The Commons Strateg Group. Repéré à http://patternsofcommoning.org/we-are-one-bigconversation-commoning-in-venezuela/

Chartier, D. et Le Crosnier, H. (2015) Acter la fin d'un monde pour activer l'alternative. ONG et acteurs de l'altermondialisme à Rio+20. Dans J. Foyer (dir.), Regards croisés sur Rio+20 (p. 281-304). Paris, France: CNRS Éditions.

Cillero, M. (2016, $1^{\text {er }}$ janvier). ¿En qué consiste el municipalismo y por qué gana peso en la política española? UnitedExplanations. Repéré à http://www.unitedexplanations.org/2016/01/01/que-es-elmunicipalismo/

Combis-Schlumberger, H. (2015, 18 mars). Suite à un Instagram de Fleur Pellerin, le musée d'Orsay autorise la photo. France Culture. Repéré à

https://www.franceculture.fr/sociologie/suite-un-instagram-de-fleurpellerin-le-musee-dorsay-autorise-la-photo 
Communa di Bologne (2013). Regolamento sulla collaborazione per la cura e rigenerazione dei beni comuni urbani. Repéré à

http://www.comune.bologna.it/sites/default/files/documenti/REGOL AMENTO\%20BENI\%20COMUNI.pdf

Communia. (2010). The Public Domain Manifesto. Repéré à https://publicdomainmanifesto.org

Cornu-Volatron, M., Orsi, F. et Rochfeld, J. (dir.), (2017). Dictionnaire des biens communs. Paris, France: Presses universitaires de France.

Dafermos, G. (2017). The Catalan Integral Cooperative: An Organizational Study of a Post-Capitalist Cooperative. Repéré à http://commonstransition.org/the-catalan-integralcooperative-an-organizational-study-of-a-post-capitalistcooperative/

Dardot P. et Laval, C. 2014. Commun : essai sur la révolution au XXI siècle. Paris, France : La Découverte.

Dulong de Rosnay, M. et Le Crosnier, H. (2012). An Introduction to the Digital Commons: From Common-Pool Resources to Community Governance. Dans T. Dedeurwaerdere (dir.), Building Institutions for Sustainable Scientific, Cultural and genetic Resources Commons. Louvain-la-Neuve, Belgique : International Association for the Study of the Commons. Repéré à https://halshs.archivesouvertes.fr/halshs-00736920

Eghbal, N. (2017). Roads and Bridges: The Unseen Labor Behind Our Digital Infrastructure. Ford Foundation. Repéré à https://www.fordfoundation.org/library/reports-and-studies/roadsand-bridges-the-unseen-labor-behind-our-digital-infrastructure

Festa, D. (2016). Les communs urbains. L'invention du commun. Tracés, (16), 233-256. Repéré à https://journals.openedition.org/traces/6636\#text

Greer, A. (2015, 31 mars). Confusion sur les communs. La vie des idées. Repéré à http://www.laviedesidees.fr/Confusion-surles-Communs.html

Hardin, G. (1968). The Tragedy of the Commons. Science, 162(3859), 1243-1248. Repéré à

http://www.sciencemag.org/content/162/3859/1243.full 
Heller, M. (1998). The Tragedy of the Anticommons: Property in the Transition From Marx to Markets. Harvard Law Review, 111(3), 621-688. Repéré à https://repository.law.umich.edu/cgi/viewcontent.cgi?referer=\&http sredir $=1 \&$ article $=1608 \&$ context $=$ articles

Hess, C et Ostrom, E. (dir.). 2007. Understanding Knowledge as a Commons: From Theory to Practice. Cambridge, MA : MIT Press.

Hess, C. (2011). Inscrire les communs de la connaissance dans les priorités de recherche. Dans VECAM (dir.) Libres Savoirs: les biens communs de la connaissance. Caen, France : C\&F éditions. Repéré à https://vecam.org/archives/article1307.html

Kamp, P.-H. (2014). Quality Software Costs Money Heartbleed Was Free: How to Generate Funding for FOSS. ACM Queue, 12(6). Repéré à https://queue.acm.org/detail.cfm?id=2636165

Leaf, C. (2005, 19 septembre). The Law of Unintended Consequences. Fortune Magazine. Repéré à http://archive.fortune.com/magazines/fortune/fortune_archive/2005 109/19/8272884/index.htm

Le Crosnier, H. (2011a). Subtle But Effective: Modern Forms of Enclosures. Dans D. Bollier et S. Helfrich (dir.), The Wealth of the Commons (p. 177-181). Amherst, MA : Levellers Press.

Le Crosnier, H. (2011b, 3 juillet). Une bonne nouvelle pour la théorie des communs. Vacarme, (56). Repéré à https://vacarme.org/article2067.html (Repris dans Le Crosnier, 2015).

Le Crosnier H., Neubauer C. et Storup B. (2013). Sciences participatives ou ingénierie sociale: quand amateurs et chercheurs co-produisent les savoirs. Hermès, 3(67), 68-74. Repéré à https://www.cairn.info/revue-hermes-la-revue-2013-3page-68.htm

Le Roy, E. (2015). How I Have Been Conducting Research on the Commons for Thirty Years Without Knowing It. Dans D. Bollier et S. Helfrich, S. (dir.), Patterns of Commoning, Amherst, MA: The Commons Strategy Groug. Repéré à 
http://patternsofcommoning.org/how-i-have-been-conductingresearch-on-the-commons-for-thirty-years-without-knowing-it/

Lessig, L. (2004). Free Culture: How Big Media Use Technology and the Law to Lock Down Culture and Control Creativity. New York, NY: The Penguin Press. Repéré à http://www.free-culture.cc/freeculture.pdf

Lévêque, F. et Ménière, Y. (2003). Économie de la propriété intellectuelle. Paris, France : La Découverte.

Lucarelli, A. (2018). Biens communs. Contribution à une théorie juridique. Droit et société, 98(1), 141-157. Repéré à https://www.cairn.info/revue-droit-et-societe-2018-1-page-141.htm

Mazzone, J. (2011). Copyfraud and Other Abuses of Intellectual Property Law. Mountain View, CA: Stanford University Press.

Nature (2006). Nature's peer review debate. Nature. Special Web Edition. Repéré à https://www.nature.com/nature/peerreview/debate/index.html

Orsi, F. (2013). Elinor Ostrom et les faisceaux de droits: l'ouverture d'un nouvel espace pour penser la propriété commune. Revue de la Régulation, 2(14). Repéré à http://regulation.revues.org/10471

Ostrom, E. (1990). Governing the Commons: The Evolution of Institutions for Collective Action. Cambridge, MA : Cambridge University Press.

Ostrom, E. (2009). A Polycentric Approach for Coping With Climate Change. Policy Research Paper, (5095). Repéré à https://elibrary.worldbank.org/doi/abs/10.1596/1813-9450-5095

Peugeot, V. (2016, septembre). Facilitatrice, protectrice, instituante, contributrice: la loi et les communs. Communication présentée au Colloque de Cerisy «Vers une république des biens communs?". Repéré à https://vecam.org/Facilitatrice-protectrice-instituante-contributricela-loi-et-les

Rey, A. (2011). La guerre des communs. Dans VECAM (dir.), Libres savoirs: les biens communs de la connaissance (p. 347-351). Caen, France : C\&F éditions. 
Une introduction aux communs de la connaissance

Samuelson, P. A. (1954). The Pure Theory of Public Expenditures. Review of Economics and Statistic, 36(4), 387389.

SavoirCom1 (2018). Calendrier de l'avent du domaine public. Repéré à http://aventdudomainepublic.org

VECAM (dir.). (2011). Libres savoirs: les biens communs de la connaissance. Caen, France : C\&F éditions.

Weiler, N. (2016, 11 octobre). Un bien commun sauvé du béton et créateur d'emplois: les marais salants de Guérande. Bastamag. Repéré à https://www.bastamag.net/Un-biencommun-sauve-du-beton-et-createur-d-emplois-les-maraissalants-de 